Graphical Abstract

\title{
Effects of hysteresis window on con- tact angle hysteresis behaviour at large Bond number
}

Jiapei Yang, Xiao Ma, Linlin Fei, Xiaoqing Zhang, Kai H. Luo, Shijin Shuai
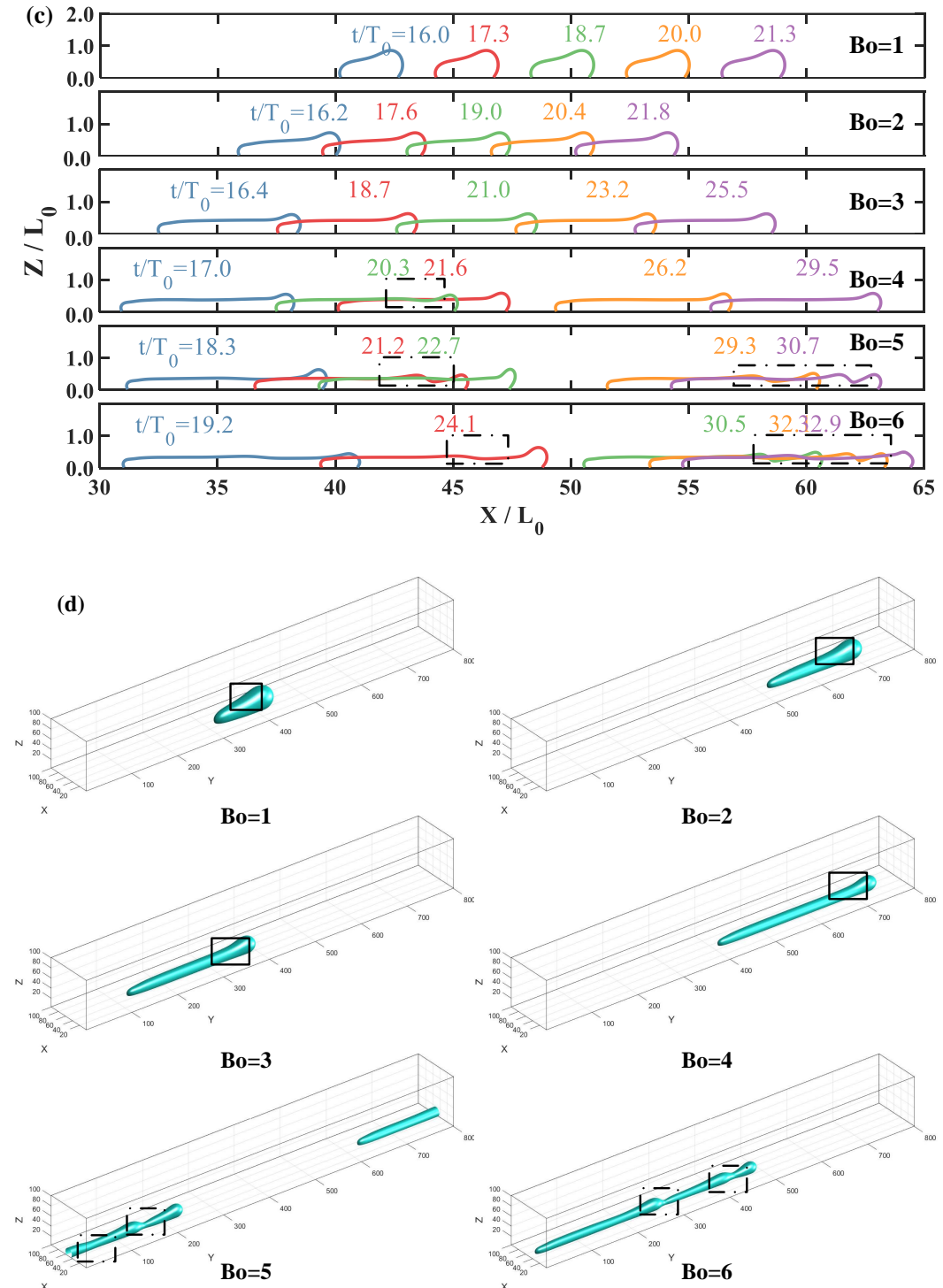
Highlights

\section{Effects of hysteresis window on con- tact angle hysteresis behaviour at large Bond number}

Jiapei Yang, Xiao Ma, Linlin Fei, Xiaoqing Zhang, Kai H. Luo, Shijin Shuai

- A modified pseudo-potential LB multiphase model with tunable surface tension is proposed, which is further coupled with the geometrical formulation contact angle scheme to investigate the motion of droplets invoking the contact angle hysteresis.

- The droplet morphology change is investigated in the two-dimensional parameter space, i.e., the Bond number and hysteresis window.

- The droplet oscillation phenomenon at large Bond numbers at the equilibrium stage is observed for the first time.

- The phase diagram of droplet oscillation with varying Bond number, receding angle, and advancing angle is obtained. 


\title{
Effects of hysteresis window on contact angle hysteresis behaviour at large Bond number
}

\author{
Jiapei Yang ${ }^{\mathrm{a}}$, Xiao Ma ${ }^{\mathrm{a}}$, Linlin Fei ${ }^{\mathrm{b}}$, Xiaoqing Zhang ${ }^{\mathrm{a}}$, Kai H. Luo ${ }^{\mathrm{c}, *}$, Shijin \\ Shuai ${ }^{\mathrm{a}}$ \\ ${ }^{a}$ State Key Laboratory of Automotive Safety and Energy, School of Vehicle and Mobility, \\ Tsinghua University, Beijing 100084, China \\ ${ }^{b}$ Center for Combustion Energy, Key Laboratory for Thermal Science and Power \\ Engineering of Ministry of Education, Department of Energy and Power Engineering, \\ Tsinghua University, Beijing 100084, China \\ ${ }^{c}$ Department of Mechanical Engineering, University College London, Torrington Place, \\ London WC1E \%JE, UK
}

\begin{abstract}
Contact angle hysteresis, defined as the difference between advancing and receding contact angles, is an important phenomenon in multiphase flow on a wetting surface. In this study, a modified pseudo-potential lattice Boltzmann (LB) multiphase model with tunable surface tension is proposed, which is further coupled with the geometrical formulation contact angle scheme to investigate the motion of droplets invoking the contact angle hysteresis. We focus on the dynamic behaviour of droplets driven by a body force at the Bond number ranging from 1 to 6 , which is defined as the ratio of the body force to the capillary force. The droplet morphology change is examined by varying (i) the Bond number and (ii) the hysteresis window. Results show the droplet morphology evolution can be classified into different stages, including stretch, relaxation, and equilibrium. The droplet oscillation phenomenon at large Bond numbers at the equilibrium stage is observed for the first time. In addition, it is found that such oscillation can lead to the breakup and/or coalescence of droplets when the surface waves spread on the top of the droplet. Furthermore, there is slight oscillation of the normalized length, width and height at the equilibrium stage for the neutral hysteresis window
\end{abstract}

\footnotetext{
*Corresponding author.

Email address: K.Luo@ucl.ac.uk (Kai H. Luo)
} 
while more dramatic oscillation will appear for the hydrophobic hysteresis window.

Keywords: lattice Boltzmann method, contact angle hysteresis, multiphase flow, Bond number

\section{Introduction}

The dynamic behaviour of water droplets on a solid surface is a ubiquitous phenomenon in daily life, such as raindrops on glass panes and industrial applications, such as in droplet cooling of gas turbine blades, spray coating, and droplet sliding in flow channels of proton exchange membrane fuel cells, and so on. Among the diverse phenomena of droplet dynamics, contact angle hysteresis is a critical problem, which is far from being fully solved. When a droplet moves on a flat surface, the droplet shape will be changed and the contact angle at the contact line falls in between the maximum (advancing) and minimum (receding) values [1]. Here, the difference between the advancing and receding contact angles is defined as contact angle hysteresis [2]. One cannot accurately capture droplet deformation and adhesion force without considering contact angle hysteresis [3]. The contact angle hysteresis can cause an additional pressure drop in mini and micro channels [4, 5]. In the simulation of the slug flow, the contact angle hysteresis can be attributed to the slug elongation and the post-detachment instability [6]. Hence, it is of essential significance to study the effects and mechanisms of contact angle hysteresis.

Considerable efforts have been devoted to model and study contact angle hysteresis. Zhang and Qin [7] proposed a theoretical method of correlations for contact angle hysteresis through force balance for a moving droplet on a hydrophobic surface. A single parameter model, which depended on the fractal structure of the contact line was derived by $\mathrm{Wu}$ and $\mathrm{Ma}$ [8] to describe the connection among the equilibrium, receding and advancing contact angles. With the rapid development of the computing technology, it is more and more efficient to explore the phenomenon of contact angle hysteresis by numerical experiments. The large-scale molecular dynamics (MD) was used by Fernández-Toledano et al. [9] to simulate a nano droplet moving across a solid surface driven by an external force. A correlation between the contact angle hysteresis and the velocity of the contact line was coupled in a volume of fluid (VOF) method by adding an uncompensated force in Young's 
equation to simulate the droplet deformation with contact angle hysteresis on a surface by Theodorakakos et al. [10]. Similarly, Santos and White [11 developed a contact angle hysteresis model by adding a friction force in Young's equation based on the hysteresis window rather than the contact line velocity, which was then solved by a finite-elements method. A geometrical formulation of wetting condition for diffuse-interface simulations is proposed by Ding et al. [12] and used to investigate a 3D sliding droplet in shear flows. Ba et al. [13] introduced a numerical method based macroscopic contact angle to their multiphase model to simulate the droplet motion in shear flow.

Recently, the lattice Boltzmann method (LBM) has been widely adopted for numerical simulations of complex fluid flows [14, 15, 16, 17]. For the study of multiphase flows with contact angle hysteresis, Wang et al. [18] used the He-Chen-Zhang [19] and Lee-Lin [20] LBM model incorporated with geometrical boundary conditions proposed by Ding et al. [12] to study the motion of a droplet driven by a shear flow for varying hysteresis window. Related simulations were carried out by Liu et al. [21], which adopted the color-fluid LBM model coupled with geometrical wetting boundary conditions to simulate the behaviour of contact angle hysteresis for a wide range of kinematic viscosity ratios. But the density ratio was not large enough to represent a water-air system.

In the past, many researchers focused on the motion of droplets under a body force [18, 22, 23, 24, 25]. Semprebon et al. [23] adopted an energy minimization method to describe the onset of motion of a droplet driven by a body force with contact angle hysteresis. In their simulations, the Bond number, which is defined as the ratio of the body force to the capillary force, is in the range of $0.0-1.6$, that can include a critical value for the transition from pinned to depinned state for the droplet. In Refs. [18, 24], the Bond number is also set to be less than 2.0 to simulate a droplet on a tilted wall. A open source software, Surface Evolver, based on the principle of minimization of energy and conservation of volume subject to constraints was used by Janardan et al. [25] to obtain the effect of initial conditions on the shape of a droplet on an inclined plane, which was on the threshold of sliding.

However, there is a big gap in the study of droplets with contact angle hysteresis driven by an external force at high Bond numbers (>2.0). Besides droplets on a vertical plane, a high Bond number may be present in situations like curtain coating [26], droplets on a horizontal substrate with acceleration 
(e.g., a rotating disk [27, 28]), and droplets with electrowetting [29, 30].

In this study, a modified pseudo-potential multiphase model with tunable surface tension and the geometrical formulation scheme is developed to simulate contact angle hysteresis. We focus on the dynamic behaviour of droplets with contact angle hysteresis at high Bond numbers from 1 to 6 . This paper is organized as follows. We start with the description of the pseudo-potential multiphase model and geometrical wetting boundary condition. Then we compare our LBM results with those of Semprebon et al. [23] and verify the independence of the system setup. Next the droplet dynamic behaviours in respond to (i) varying Bond number and (ii) varying hysteresis window are examined. Finally, the conclusion of this study is given.

\section{Numerical method}

\subsection{The multiple-relaxation-time LB model}

A three-dimensional LB model with a multiple-relaxation-time (MRT) collision operator based on D3Q19 discrete velocity model is adapted to simulate the dynamics of a liquid droplet. For the details of the D3Q19 discrete velocity model, the interested readers are kindly directed to [31, 32, 33]. The evolution of the particle distribution function $f_{i}$ can be written as

$\bar{f}_{i}\left(\mathbf{x}+\mathbf{e}_{i} \Delta t, t+\Delta t\right)=\bar{f}_{i}(\mathbf{x}, t)-\left.\Lambda_{i, k}\left[\bar{f}_{k}-f_{k}^{e q}\right]\right|_{(\mathbf{x}, t)}+\left(\mathbf{I}-\frac{\Lambda_{i, k}}{2}\right) \bar{F}_{i}(\mathbf{x}, t) \Delta t$

where $\mathbf{x}$ is the position vector, $t$ is time, $\mathbf{e}_{i}$ is the discrete velocity vector, $\Delta t$ is the lattice time step, $\bar{f}_{i}=f_{i}-\Delta t \bar{F}_{i} / 2, f_{i}^{e q}$ is the corresponding equilibrium distribution function, $\mathbf{I}$ is the unit tensor matrix, and $\Lambda_{i, k}=\left(\mathbf{M}^{-\mathbf{1}} \mathbf{S M}\right)_{i, k}$. The forcing terms $\bar{F}_{i}$ read $[34$

$$
\bar{F}_{i}=\omega\left(\left|\mathbf{e}_{i}\right|^{2}\right)\left[\frac{\mathbf{e}_{i}-\mathbf{u}}{c_{s}^{2}}+\frac{\left(\mathbf{u} \cdot \mathbf{e}_{i}\right) \mathbf{e}_{i}}{c_{s}^{4}}\right] \cdot \mathbf{F}
$$

where the weighting factors are $\omega(1)=1 / 6$ and $\omega(2)=1 / 12, \mathbf{u}$ is the fluid velocity, $c_{s}=1 / \sqrt{3}$ is the lattice sound speed, and $\mathbf{F}$ is the total force including the pseudopotential interaction force and any body force

$$
\mathbf{F}=\mathbf{F}_{\text {int }}+\mathbf{F}_{g}
$$

The diagonal matrix $\mathbf{S}$ specifying the relaxation rates is given by

$$
\mathbf{S}=\operatorname{diag}\left(s_{0}, s_{e}, s_{\varepsilon}, s_{0}, s_{q}, s_{0}, s_{q}, s_{0}, s_{q}, s_{v}, s_{\pi}, s_{v}, s_{\pi}, s_{v}, s_{v}, s_{v}, s_{t}, s_{t}, s_{t}\right)
$$


where $s_{v}$ is related to the kinematic viscosity through $\nu=\left(1 / s_{v}-1 / 2\right) c_{s}^{2}$. In this work, we use $\nu_{l}=0.01$ and $\nu_{g}=0.1497$ for liquid and gas fluids, respectively, the ratio of which corresponds to the realistic kinematic viscosity ratio $(1: 14.97)$ for the water-air system at $20{ }^{\circ} \mathrm{C}$.

Through the transformation matrix $\mathbf{M}$, the collision process in physical space of Eq. (11) can be transformed into the moment space

$$
\overline{\mathbf{m}}^{*}=\overline{\mathbf{m}}-\mathbf{S}\left(\overline{\mathbf{m}}-\overline{\mathbf{m}}^{e q}\right)+\left(\mathbf{I}-\frac{\mathbf{S}}{2}\right) \Delta t \widetilde{\mathbf{F}}
$$

where $\overline{\mathbf{m}}=\mathbf{M f}, \mathbf{m}^{e q}=\mathbf{M f}{ }^{e q}$, and $\widetilde{\mathbf{F}}=\mathbf{M} \overline{\mathbf{F}}$.

Following the collision process, the streaming process is given as follows

$$
\bar{f}_{i}\left(\mathbf{x}+\mathbf{e}_{i} \Delta t, t+\Delta t\right)=\bar{f}_{i}^{*}(\mathbf{x}, t)
$$

where $\overline{\mathbf{f}}^{*}=\mathbf{M}^{-1} \overline{\mathbf{m}}^{*}$.

The macroscopic variables can be obtained by

$$
\rho=\sum_{i} \bar{f}_{i}, \quad \rho \mathbf{u}=\sum_{i} \bar{f}_{i} \mathbf{e}_{i}+\frac{\Delta t \mathbf{F}}{2}
$$

\subsection{The pseudopotential multiphase model}

In the pseudopotential model, a pseudopotential interaction force is introduced to describe the interaction among fluid particles, which makes it easy to realize liquid-gas phase transition [35, 36]. For the single-component model, this interaction force is defined as

$$
\mathbf{F}_{\text {int }}=-G \psi(\mathbf{x}) \sum_{i} w\left(\left|\mathbf{e}_{i}\right|^{2}\right) \psi\left(\mathbf{x}+\mathbf{e}_{i} \Delta t\right) \mathbf{e}_{i}
$$

where $G$ is the interaction strength, $\psi$ is the pseudopotential, and $w\left(\left|\mathbf{e}_{i}\right|^{2}\right)$ is the normalized weights. For the D3Q19 model, $w(1)=1 / 6, w(2)=1 / 12$.

In order to achieve high density ratio, the square-root-form pseudopotential [37] coupled with a realistic equation of state (EOS) is considered in this study

$$
\psi=\sqrt{2\left(p_{E O S}-\rho c_{s}^{2}\right) / G c^{2}}
$$

where $p_{E O S}$ is the pressure determined by the EOS. The EOS is selected as a piecewise linear equation of state as, which is given by [38]

$$
p(\rho)=\left\{\begin{array}{l}
\rho \theta_{v}, \rho \leq \rho_{1} \\
\rho_{1} \theta_{v}+\left(\rho-\rho_{1}\right) \theta_{m}, \quad \rho_{1} \leq \rho \leq \rho_{2} \\
\rho_{1} \theta_{v}+\left(\rho_{2}-\rho_{1}\right) \theta_{m}+\left(\rho-\rho_{2}\right) \theta_{l}, \quad \rho>\rho_{2}
\end{array}\right.
$$


where $\theta_{v}, \theta_{l}$ and $\theta_{m}$ are adjustable parameters of slopes in different regions. The other unknown variables $\rho_{1}$ and $\rho_{2}$ can be solved according to the following two equations

$$
\begin{gathered}
\int_{\rho_{v}}^{\rho_{l}}\left(\rho_{1}-\rho_{v}\right) \theta_{v}+\left(\rho_{2}-\rho_{1}\right) \theta_{m}+\left(\rho_{l}-\rho_{2}\right) \theta_{l}=0 \\
\int_{\rho_{v}}^{\rho_{l}} \frac{1}{\rho} d p=\log \left(\rho_{1} / \rho_{v}\right) \theta_{v}+\log \left(\rho_{2} / \rho_{1}\right) \theta_{m}+\log \left(\rho_{l} / \rho_{2}\right) \theta_{l}=0
\end{gathered}
$$

where $\rho_{l}$ and $\rho_{v}$ represent the liquid and vapour coexistence densities, respectively.

Here, we focus on a high density ratio with $\rho_{l} / \rho_{v}=1 / 0.001=1000$, which is a typical ratio for the water-air system at around $20{ }^{\circ} \mathrm{C}$. The values of $\theta_{v}, \theta_{l}$ and $\theta_{m}$ are set to be

$$
\theta_{v}=c_{s}^{2} / 2, \quad \theta_{l}=c_{s}^{2}, \quad \theta_{m}=-c_{s}^{2} / 40
$$

Solving Eq. (11) and Eq. (12), the parameters $\rho_{1}$ and $\rho_{2}$ are obtained as $\rho_{1}=0.001325$ and $\rho_{2}=0.9758$.

The thermodynamic consistency is another key factor to achieve high density ratio. According to the work of [39, 40], a modified force term is proposed to achieve good thermodynamic consistency, which is given by

$$
\tilde{F}_{1}^{\prime}=\tilde{F}_{1}+\frac{114 \varepsilon\left|\mathbf{F}_{\mathrm{int}}\right|^{2}}{\psi^{2}\left(1 / s_{1}-0.5\right)}
$$

where $\varepsilon$ is an adjustable parameter to control the mechanical stability condition. In this work, $\varepsilon$ is fixed as 0.1 .

To achieve the tunable surface tension in the Shan-Chen-type LB model, a correction term can be added on the right hand of the Eq. (5) [41], i.e.,

$$
\overline{\mathbf{m}}^{*}=\overline{\mathbf{m}}-\mathbf{S}\left(\overline{\mathbf{m}}-\overline{\mathbf{m}}^{e q}\right)+\left(\mathbf{I}-\frac{\mathbf{S}}{2}\right) \Delta t \widetilde{\mathbf{F}}+\Delta t C
$$

Inspired by the original correction formulation proposed in $2 \mathrm{D}$ model by Li et al. [41], here we propose to add the following correction term $\mathrm{C}$ to tune 
the surface tension in the D3Q19 lattice:

$$
C=\left\{\begin{array}{c}
0 \\
\frac{76}{5} s_{v}\left(Q_{x x}+Q_{y y}+Q_{z z}\right) \\
0 \\
0 \\
0 \\
0 \\
0 \\
0 \\
0 \\
-s_{v}\left(2 Q_{x x}-Q_{y y}-Q_{z z}\right) \\
0 \\
-s_{v}\left(Q_{y y}-Q_{z z}\right) \\
0 \\
-s_{v} Q_{x y} \\
-s_{v} Q_{y z} \\
-s_{v} Q_{x z} \\
0 \\
0 \\
0
\end{array}\right.
$$

The components of tensor Q can be derived from

$$
Q=\kappa \frac{G}{2} \psi(x) \sum_{i} w\left(\left|\mathbf{e}_{i}\right|^{2}\right)\left[\psi\left(\mathbf{x}+\mathbf{e}_{i} \Delta t\right)-\psi(\mathbf{x})\right] \mathbf{e}_{i} \mathbf{e}_{i}
$$

where $\kappa$ is an adjustment factor of surface tension.

\subsection{Boundary conditions}

The periodic boundary condition is applied in the $\mathrm{X}$-axis and $\mathrm{Y}$-axis directions. And the top is non-slip boundary condition. Next we consider the implementation of contact angle hysteresis. It is well known that there are various schemes of wettability boundary conditions including densitybased contact angle scheme [42, 43], pseudopotential-based contact angle scheme [44, 45], and modified pseudopotential-based contact angle scheme [46]. These schemes achieve a prescribed contact angle through modelling the force between the fluid and solid phases. However, these types of scheme can not simulate the contact angle hysteresis. Thanks to the work of Ding 
et al. [12], the geometrical formulation scheme obtained from the geometry relationship of the triple contact line is proposed and performs well in prescribing contact angle hysteresis.

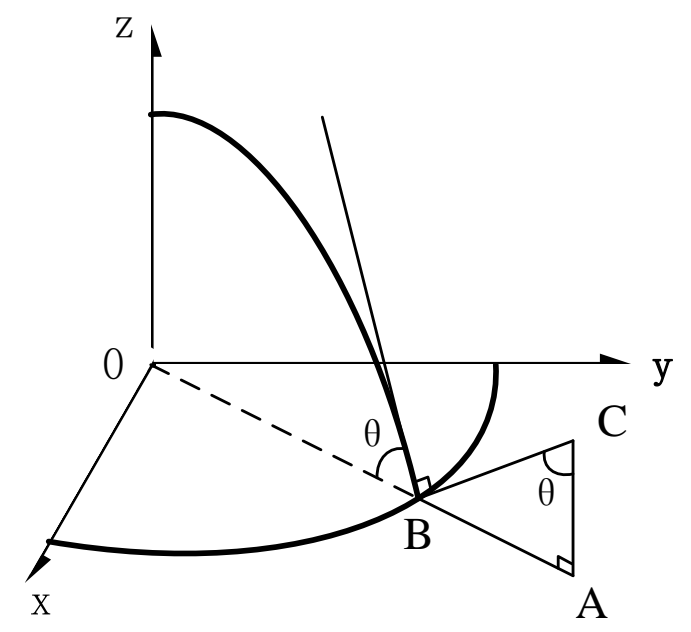

Figure 1: Schematic of the geometric relationship along the triple contact line.

Thus, the geometrical formulation scheme is adopted in our work. As shown in Fig. 1, the geometric relationship can be described by [18

$$
\tan \left(\frac{\pi}{2}-\theta\right)=\frac{\overline{A C}}{\overline{A B}}=-\frac{\partial \rho}{\partial z} / \sqrt{\left(\frac{\partial \rho}{\partial x}\right)^{2}+\left(\frac{\partial \rho}{\partial y}\right)^{2}}
$$

Then, the approximate derivative can be calculated using difference quotient:

$$
\rho_{i, j, 0}=\rho_{i, j, 2}+\tan \left(\frac{\pi}{2}-\theta\right) \xi
$$

where the subscripts represent $x, y, z$ coordinates, respectively. $\xi$ is given by [47]

$$
\xi=\sqrt{\left(\rho_{i+1, j, 1}-\rho_{i-1, j, 1}\right)^{2}+\left(\rho_{i, j+1,1}-\rho_{i, j-1,1}\right)^{2}}
$$

Any arbitrary contact angle can be imposed by assigning the value of $\theta$ in Eq. (19). Because of the existence of contact angle hysteresis, the contact line keeps still when the local contact angle $\theta$ is within a hysteresis window [24, 18] :

$$
\theta_{R} \leq \theta \leq \theta_{A}
$$


where $\theta_{R}$ and $\theta_{A}$ are the receding angle and advancing angle, respectively. If $\theta$ is geater than $\theta_{A}$, the contact line will keep going forward. On the other hand, if $\theta$ is less than $\theta_{R}$, the contact line will keep going backward. In our simulation, the contact angle hysteresis window is specified firstly and then the critical density corresponding to $\theta_{R}$ and $\theta_{A}$ is found through Eq. (19). Therefore, Eq. (21) can be rewritten as

$$
\rho_{R} \leq \rho_{i, j, 1} \leq \rho_{A}
$$

After that, let us compare $\rho_{i, j, 1}$ with $\rho_{R}$ and $\rho_{A}$.

$$
\bar{\rho}_{i, j, 1}=\left\{\begin{array}{l}
\rho_{A}, \rho_{i, j, 1}>\rho_{A} \\
\rho_{i, j, 1}, \quad \rho_{R} \leq \rho_{i, j, 1} \leq \rho_{A} \\
\rho_{R}, \rho_{i, j, 1}<\rho_{R}
\end{array}\right.
$$

Based on the model as described above, contact angle hysteresis can be realized easily, whatever the source of hysteresis is.

\section{Model validation}

\subsection{Model validation}

It is necessary to valid the model proposed in this paper. The YoungLaplace law validation has been done in our previous work [48, 49]. However, in order to derive the value of surface tension, three-dimensional static droplet

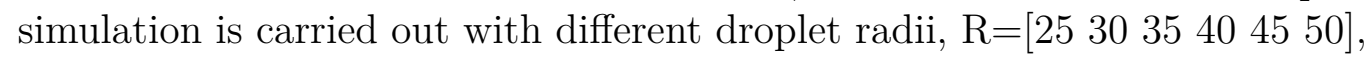
in a cubic domain of $200 \times 200 \times 200$. According to the Young-Laplace law, $\Delta p=2 \gamma / R$, surface tension $\gamma$ at $\kappa=0.0$ can be derived from the slope of the line as shown in Fig. 2.

After that, we compare our LBM simulation results with the simulation results of the energy minimization method (by minimising both free interface and gravitational energies) [23]. The comparisons of temporal evolution of length/width ratio, side-view and top-view of droplet contours at the initial contact angle $\theta_{\text {in }}=72^{\circ}$ and Bond number $B o=1.85$ are presented in Figs. 3(a)(b)and(c), respectively. From the graphs we can see that the LBM simulation results concur well with the results of the energy minimization method. 


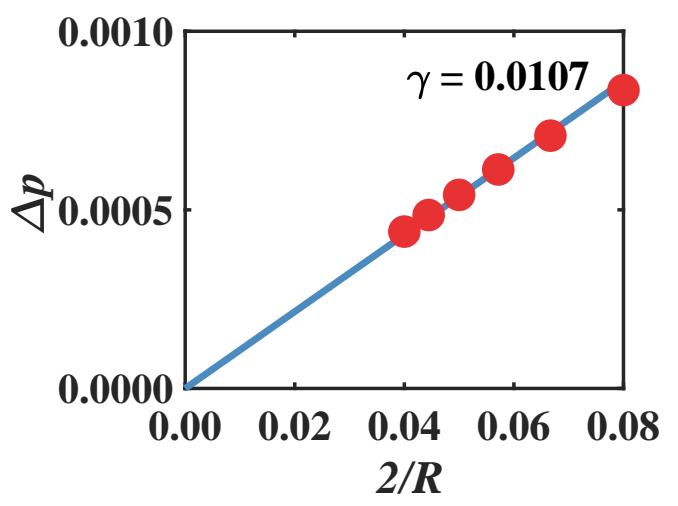

Figure 2: The Young-Laplace law validation based on the static droplet simulation.

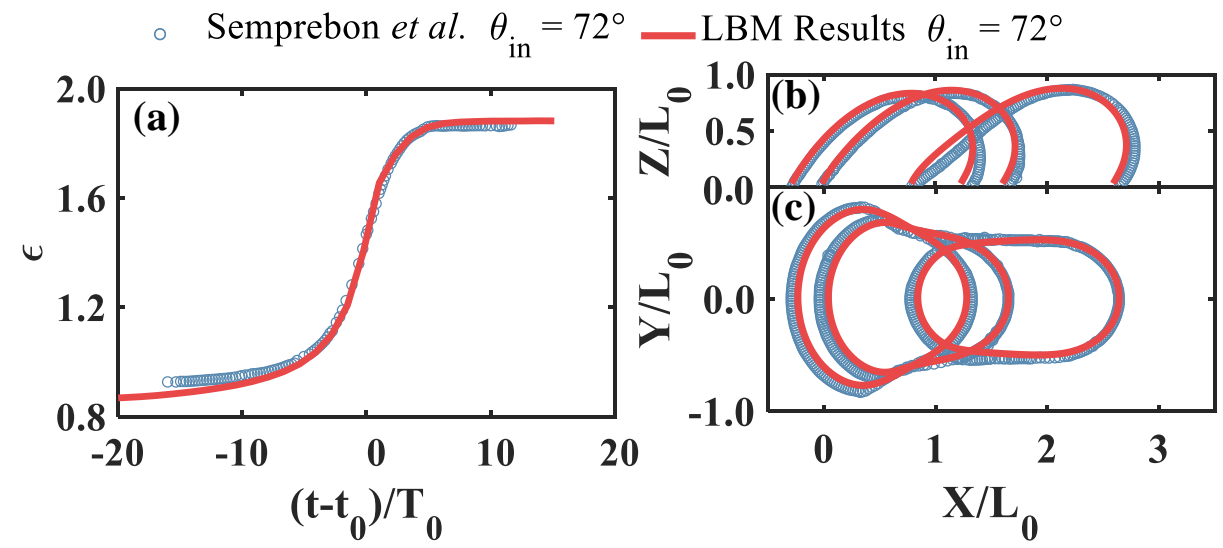

Figure 3: Validation of droplet contact angle hysteresis model with the results of Semprebon et al. [23]. (a) temporal evolution of length/width ratio. (b) and (c) side-view and top-view of droplet contours, respectively

\subsection{Independence validation}

The independence analysis of the mesh, droplet initial radius and droplet equilibrium contact angle (or droplet initial contact angle) is carried out to guarantee a parameter independent results. The detailed parameter setup of independence analysis at $B o=1.0, \theta_{A}=105^{\circ}, \theta_{R}=75^{\circ}$, are shown in Table 1. Here we choose contact angle and length/width ratio as indicators. It can be seen that radius $=30$, mesh $100 \times 100 \times 800, \theta_{\text {eq }}=0.5 \times\left(\theta_{A}+\theta_{R}\right)$ are enough for independent solution as portrayed by Fig. 4 . 
Table 1: Independence analysis at $B o=1.0, \theta_{A}=105^{\circ}, \theta_{R}=75^{\circ}$.

\begin{tabular}{ccc}
\hline Radius & Equilibrium contact angle & Mesh size \\
\hline 30 & 90 & $100 \times 100 \times 800$ \\
35 & 90 & $100 \times 100 \times 800$ \\
30 & 90 & $200 \times 200 \times 1000$ \\
30 & 82.5 & $100 \times 100 \times 800$ \\
30 & 97.5 & $100 \times 100 \times 800$ \\
\hline
\end{tabular}

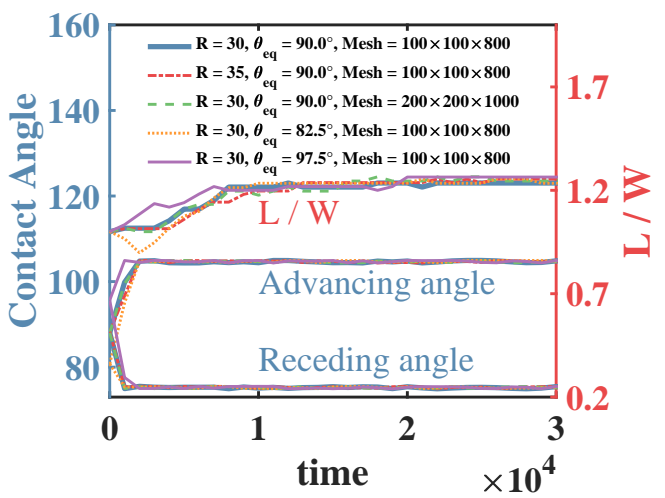

Figure 4: Independence analysis of the mesh, droplet initial radius and droplet equilibrium contact angle based on temporal evolution of advancing angle, receding angle, and length/width ratio.

\subsection{Physical problem}

To simplify the problem, a drop on a plane substrate with a horizontal driving body force $F_{g}$ is considered, as shown in Fig. 5. This condition can represent droplets on a vertical plane [23] or on a horizontal plane with acceleration. Here we define the body force $F_{g}$ in Eq. 16 as

$$
F_{g}=\left(\rho-\rho_{g}\right) g
$$

where $g$ is the gravitational acceleration.

Therefore, besides contact angle hysteresis window, this system is also characterized by a critical non-dimension parameter Bond number defined as the ratio of body force and surface tension, which is given by [23]

$$
B o=\frac{\rho g\left(V^{1 / 3}\right)^{2}}{\gamma}=\frac{\left(V^{1 / 3}\right)^{2}}{\left((\gamma / \rho g)^{1 / 2}\right)^{2}}=\frac{\lambda_{v}^{2}}{\lambda_{c}^{2}}
$$




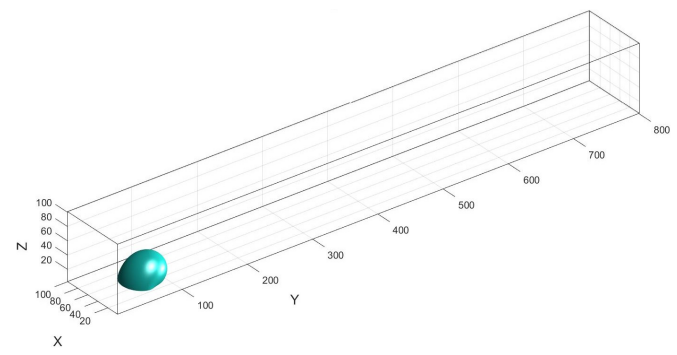

Figure 5: Schematic overview of system setup.

where $\lambda_{v}=V^{1 / 3}$ and $\lambda_{c}=(\gamma / \rho g)^{1 / 2}$ are characteristic length based on the volume and the body forces, respectively.

\section{Results and discussion}

\subsection{Bond number effect}

In this section, the dynamic behaviour of contact angle hysteresis of droplets with large Bond numbers is investigated at $\theta_{A}=130^{\circ}$ and $\theta_{R}=120^{\circ}$. In order to quantify the droplet deformation, the length scale is normalized by $L_{0}=V^{1 / 3}$ and the time scale is normalized by $t_{0}=\sqrt{L_{0} / g}$.

Figure 6 presents the morphology change of the droplet at different Bond numbers, in which (a), (b) and (c) respectively represent the time evolution of normalized length, width and height. It can be clearly seen that the normalized droplet length, width and height evolve in different stages as time progresses, including stretch, relaxation,and equilibrium. At the stretch stage, the normalized length increases while normalized width and height decrease with normalized time. Because the droplet is more susceptible to stretch along the direction of body force and shrink in other directions. Furthermore, it can be noted that after the Bond number reaches a certain value, the stretching or shrinking speeds of normalized length, width, and height almost remain unchanged at the stretch stage. This implies that the droplet stretch is mainly controlled by the body force due to the small y-component (along the direction of the body force) of the surface tension force at the stretch stage even if the $y$-component of the surface tension force gradually increases with time. And excessive stretch and shrinkage will happen with the aid of inertia.

Following the stretch stage, the relaxation stage occurs, which acts as a transition state from the stretch stage to the equilibrium stage. At this 
(1)(2)

(3)

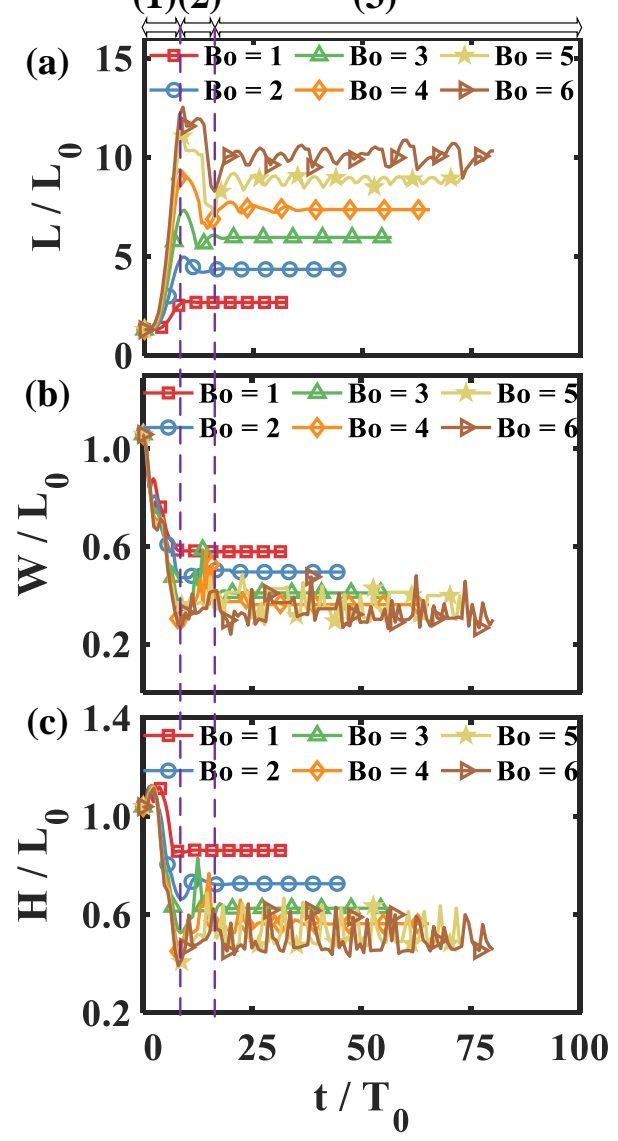

Figure 6: Morphological analysis of (a) normalized length, (b) normalized width, (c) normalized height at different Bond numbers for different stages: (1) stretch stage, (2) relaxation stage, (3) equilibrium stage.

stage, surface tension stands out from the competition with the body force. Hence the droplet gradually recovers from the maximum stretch or shrinkage in the last stage. But due to the viscous force, the normalized length is gradually weakened with normalized time, while normalized width and height are gradually enhanced with normalized time. Finally, the droplet reaches the equilibrium stage. As predicted, the normalized length, width and height almost remain constant at a small range of $\mathrm{Bo}(B o<4)$ owning to the balance of body force and surface tension. An interesting phenomenon is found that the oscillation of length, width and height starts to occur at large 
values of $\mathrm{Bo}(B o \geq 4)$. The oscillation frequency of length is much lower than that of width or height. At $B o=4$, this oscillation appears first but gradually disappears with time. And the oscillation period of droplet length at $B O=6$ is longer than that at $B o=5$, while that of width and height shows no obvious difference for both cases. However, as Bo is increased to 6 , oscillation of normalized length, width and height consistently exists with much larger oscillation amplitudes during the whole equilibrium stage. To the best of our knowledge, such an interesting phenomenon of oscillation at large Bo has not been found before.

In order to give a quantitative analysis of the relationship between the normalized shape parameters and the Bond number, we obtain the time averaged value of the normalized length, width and height with the average taken over the equilibrium stage, as shown in Fig. 7. A positive correlation between the normalized length and the Bond number while a negative correlation between the normalized width and height and the Bond number are found. This trend concurs well with the results of Semprebon et al. [23], who focused on the cases of low Bond numbers without oscillation, indicating that the time averaged value of oscillation also follows the rule of that of the non-oscillation case.

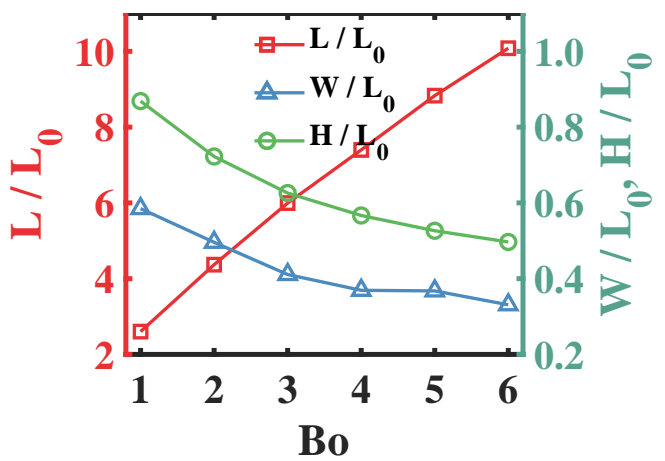

Figure 7: The time averaged value of the normalized length, width and height with the average taken over the equilibrium stage as a function of the Bond number.

The snapshots of droplet motion with varying Bond numbers for hysteresis window of $\left(120^{\circ}, 130^{\circ}\right)$ are displayed in Fig. 8, in which (a), (b), and (c) are the processes of droplet motions at the stretch, relaxation, and equilibrium stages respectively, and (d) are the isosurface images at the final normalized time step of simulation. The detailed time evolutions of the droplet are illustrated in Supplemental Videos. Due to the large given Bond 
number, the droplet soon reaches the hysteresis window and starts to move from the initial position. As expected in Fig. 8(a), the stretch stage of the deformation of all droplets at different Bond numbers can be described as a stretched droplet with a big head in the upstream side, which corresponds to the stretch stage as stated above. And we note that there is a neck (the box with a solid line) between the head and tail of droplet, which is consistent with the MD results of Fernández-Toledano et al. [9]. Subsequently, the droplet breakup (the box with a dash line) and the surface wave (the box with a dash-dotted line) appear for $B o \geq 4$ in addition to the neck at the relaxation stage as shown in Fig. 8(b). Finally, as indicated in Fig. 8(c), which corresponds to the process of droplet motion at the equilibrium stage, the droplets gradually become stable and move at a constant speed for $B o<4$ while an obvious surface wave (the box with a dash-dotted line) is observed again in the droplet for $B o \geq 4$.

The surface wave propagates from the downstream to the upstream driven by the body force, which plays a leading role in the local region of surface wave. The unavoidable deformation perpendicular to the direction of droplet motion results in oscillation of normalized width and height. Finally, surface wave coalesces into the head of the droplet. The momentum in the process of coalescence stretches the droplet length for the time being and the competition between the body force and the surface tension will make the droplet tend to be equilibrium. So the oscillation period of length depends on the average length of the droplet and the body force, which may cause the oscillation period of length for $B o=6$ to be longer than that for $B o=5$. In addition, it is now understood that the oscillation frequency difference between length and width/height is due to the fact that the length oscillation caused by the coalescence between surface wave and droplet head only happens at the end of propagation while width/height oscillation caused by the deformation can happen during the propagation process. It can also be observed that the next surface wave is successively generated at some moment of the motion of last surface wave and then spreads in the droplet. In general, the continual emergence of surface waves leads to the oscillation of normalized length, width and height. For more details, Fig. 8(d) compares the shapes of the droplets at the final normalized time for different Bond numbers. The peaks and troughs induced by surface wave (the box with a dash-dotted line) can be found on the droplet surface. And the number of peaks and troughs of droplet that simultaneously appear increases with the Bond number. 


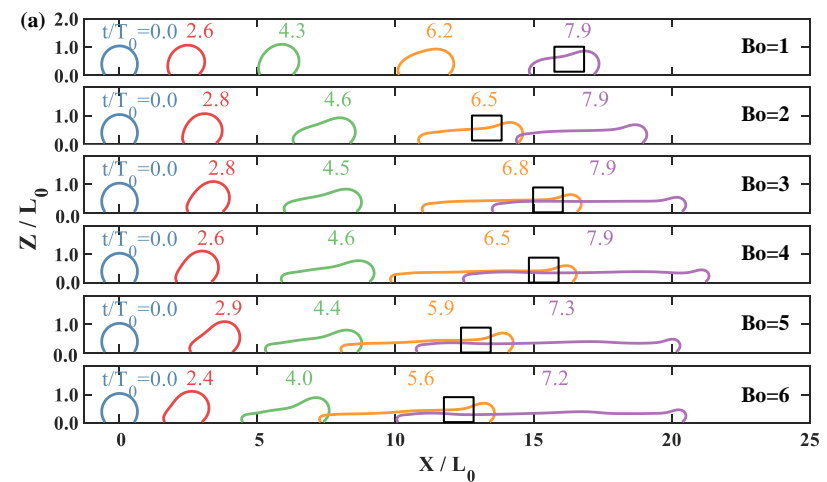

(a) The process of droplet motion at the stretch stage

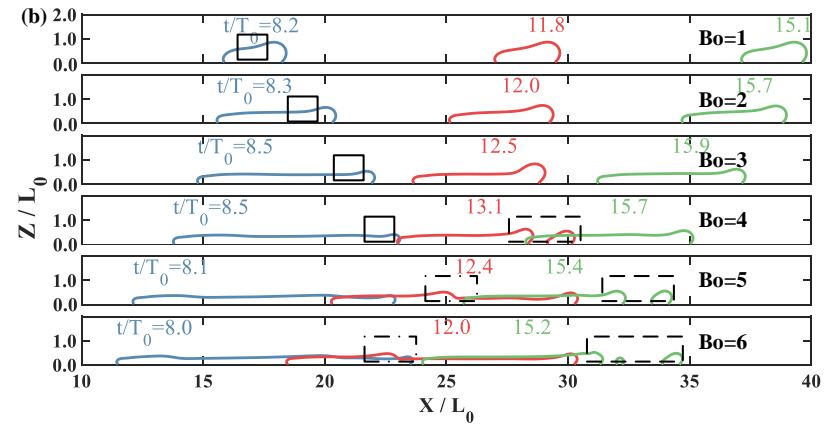

(b) The process of droplet motion at the relaxation stage

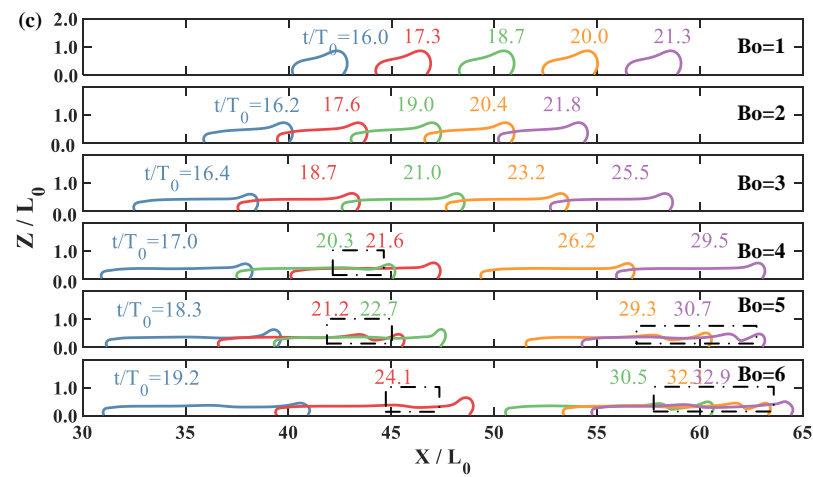

(c) The process of droplet motion at the equilibrium stage 

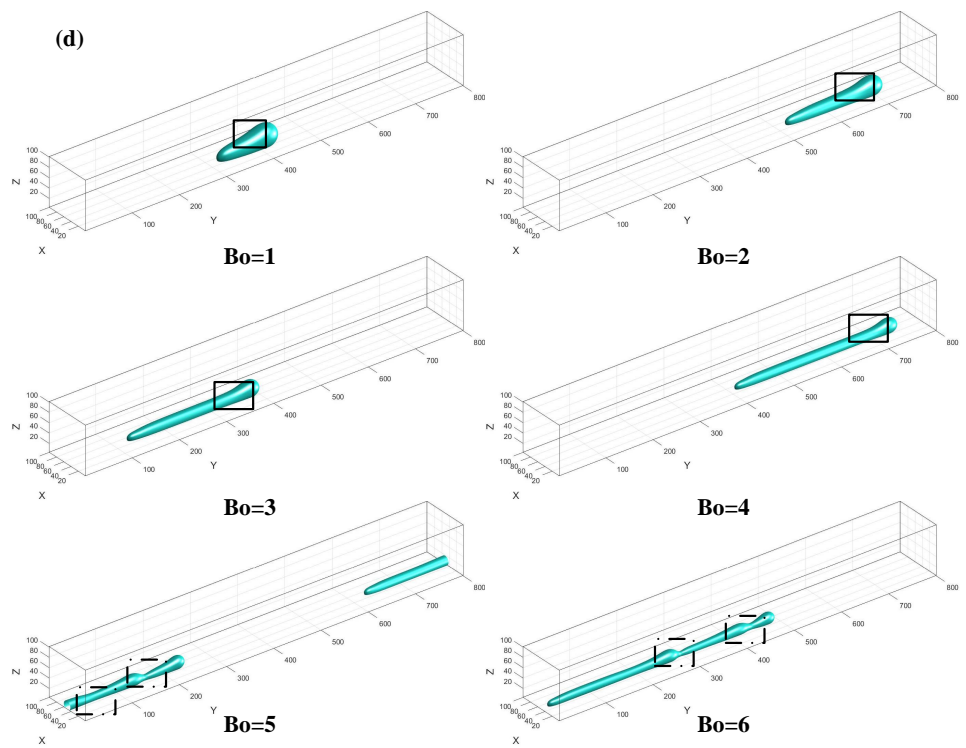

(d) The isosurface images at the final normalized time step of simulation

Figure 8: The snapshots of droplet behaviours with varying Bond numbers for hysteresis window of $\left(120^{\circ}, 130^{\circ}\right)$. The processes of droplet motions at the stretch, relaxation, and equilibrium stages are shown in (a), (b), and (c) respectively. The isosurface images at the final normalized time step of simulation are presented in (d). The box with a solid line represents neck between the head and the tail of the droplet. The box with a dash line refers to droplet breakup. The box with a dash-dotted line represents surface wave of droplet. 
To further investigate the phenomenon, we carry out a quantitative analysis of peaks and troughs induced by droplet surface waves at varying Bond numbers. Figures 9 (a) and 9(b) respectively plot changes of the number of waves that simultaneously appear and the average wavelength over the simulation time, for different Bond numbers. Apparently, the surface waves begin to appear at the relaxation stage and reach a relatively steady state at the equilibrium stage. As indicated in Fig. 9(a), there is a slightly growing tendency of the number of waves with increasing Bo although that of $B o=6$ returns to 1 intermittently. According to Fig. 9(b), with the enhancement of $\mathrm{Bo}$, the average wavelength increases slightly, which describes the distance between the peaks. This may account for the longer oscillation period of length for $B o=6$ than that for $B o=5$.

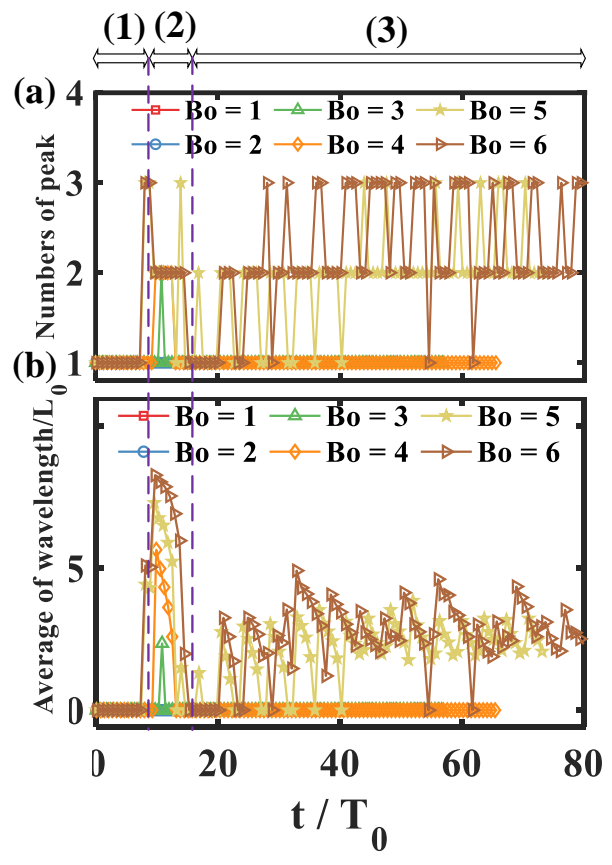

Figure 9: Quantitative analysis of peaks and troughs induced by droplet surface wave varying with Bo: (a) Number of peak; (b) Average wavelength. (1) stretch stage, (2) relaxation stage, (3) equilibrium stage.

Besides aforementioned droplet oscillation, droplet breakup is also observed in process. When the surface wave occurs, the peaks and troughs will correspondingly occur. If the local height of droplet can not meet the 
requirement of trough, the droplet will break up into several parts. Figure 10 illustrates analysis of droplet breakup with varying Bo and time evolution of normalized surface area. The normalized surface area $S / S_{0}$, where $S_{0}$ is the initial droplet surface area, is adopted to describe the droplet deformation characteristic [50] and represents a combination parameter of normalized length, width, and height of the droplet. It is noted that the tendency of the normalized surface area is similar with that of the normalized length, which means length plays an important role in the of droplet shape change. As shown in Fig. 10(a), droplet breakup firstly occurs at the relaxation stage and is concentrated at the end of the relaxation stage. At the same time of droplet breakup, there is a sharp drop in the normalized surface area. Although the breakup phenomenon occurs in the troughs of the wave, the droplet pieces merge together again quickly. Subsequently, the surface of the droplet keeps on oscillating from the downstream to the upstream. This process of breakup and merge of droplets also appears in Refs. [51, 52]. Figure 10(b) presents, in further detail, analysis of droplet breakup, including maximum number of the droplet pieces and starting time for breakup. As Bo increases, the droplet breakup is enhanced and the starting time for breakup significantly increases, which can be explained by the fact that larger Bo increases the amplitude of surface waves and reduces the average droplet height, which makes it more likely to break up in the trough of the wave.

Based on the above results, it can be concluded that this oscillation phenomenon induced by surface wave of the droplet is related to locally unbalance force, which is similar to the instability of a liquid jet of Donnelly and Glaberson [53]. The body force is exerted on mass of the droplet while the surface tension is simultaneously exerted on the contact line between the droplet and the substrate. When the droplet is stretched to a certain point, the mass distribution of the droplet will exhibit a dumbbell shape. In addition, the body force increases with the growth of the Bond number and is proportional to the mass distribution of the droplet. Thus, this perturbation makes the significance of the local body force comparable with other forces in certain regions of the droplet tail. Due to the net unbalanced force, the surface wave will appear in the droplet tail and migrate from the downstream to the upstream. After that, surface wave reaches the head of the droplet and then they merge with each other. At high Bond numbers $(B o>4)$, this process is repeated for multiple times, leading to the observed oscillation phenomenon of the droplet morphology. 
(1) (2)

(3)

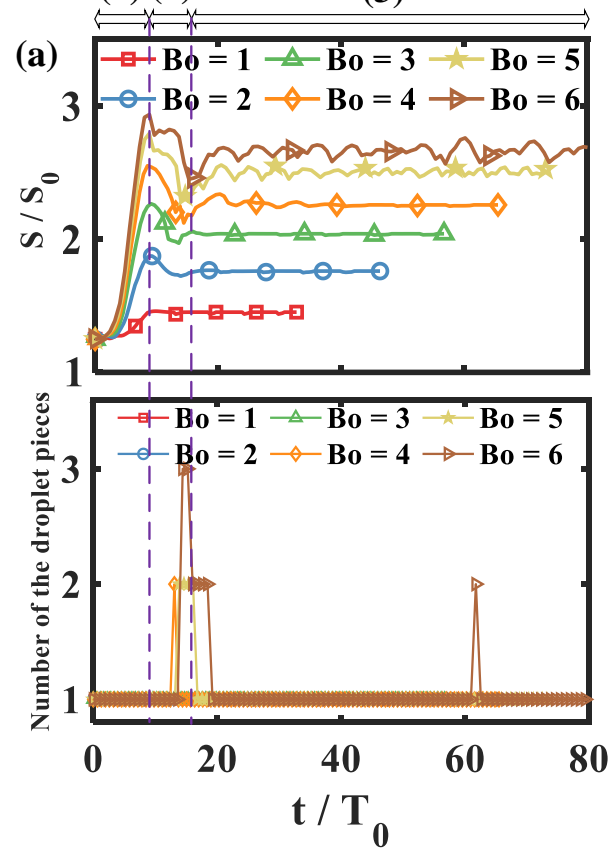

(a) Normalized surface area and number of the droplet pieces. (1) stretch stage, (2) relaxation stage, (3) equilibrium stage.

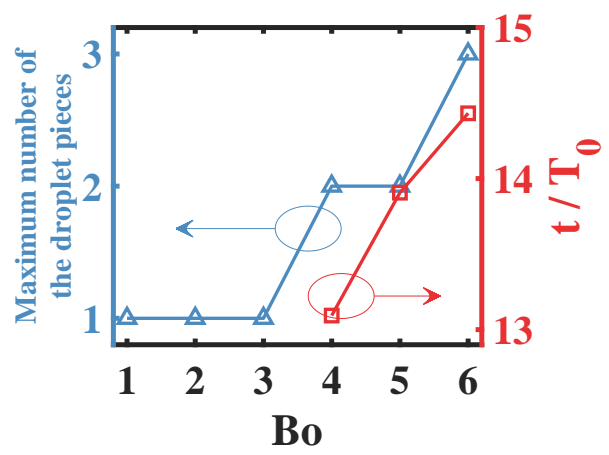

(b) Maximum number of the droplet pieces and starting time to breakup

Figure 10: Analysis of droplet breakup for varying Bo. 


\subsection{Hysteresis window effects}

Having shown the basic behaviour of droplet oscillation at large Bo, in this subsection, the effects of different hysteresis windows on oscillation phenomena are further investigated in terms of morphological analysis. Three typical scenarios including hydrophilic $\left(30^{\circ}, 60^{\circ}\right)$, neutral $\left(75^{\circ}, 105^{\circ}\right)\left(80^{\circ}, 100^{\circ}\right)$ $\left(85^{\circ}, 95^{\circ}\right)$, and hydrophobic $\left(120^{\circ}, 130^{\circ}\right)\left(120^{\circ}, 140^{\circ}\right)$ hysteresis windows are examined at $B o=5.0$. Figure 11 presents the effects of hysteresis window on morphological changes. It is noted that ratios of normalized length, width and height at the stretch stage stay almost constant with time. This also proves the droplet stretch is mainly controlled by the body force at the stretch stage as mentioned above. And the maximum stretchable or shrunken values of length, width and height at the end of the stretch stage increases with the equilibrium contact angle for all three typical hysteresis windows.

Subsequently, for the hydrophilic hysteresis window, the droplet morphology remains almost unchanged and no oscillation occurs at the equilibrium stage. For the neutral hysteresis window, there is slight oscillation of normalized length, width and height at the equilibrium stage. However, more dramatic oscillation of normalized length, width and height at the equilibrium stage appears for the hydrophobic hysteresis window. This is because the smaller the contact angle, the lower the center of gravity of the droplet, which makes droplet more stable.

In addition, the results of Figs. 11(d) - 11(i) show that the oscillation amplitude of morphological changes for neutral and hydrophobic hysteresis windows become more intensive with increasing width of the hysteresis window. We think that the difference between the upstream and the downstream hysteresis windows acts like a "container" for the droplet mass. And the non-uniform mass distribution of the droplet and droplet deformation will be enhanced as that difference is enlarged, which leads to more dramatic oscillation.

The surface area and analysis of droplet breakup with neutral and hydrophobic hysteresis windows at $B o=5.0$ are presented in Fig. 12. It is clear to see that the droplets for all hysteresis windows exhibit a breakup phenomenon and the breakup time almost starts from the beginning of the relaxation stage. However, the number of droplet pieces for breakup almost stay a steady level in the neutral case while the number of droplet pieces for breakup is oscillating in the hydrophobic case, which means the droplet is in a state of alternate breakup-coalescence. A possible explanation for such results might be the oscillation of droplet length induced by surface 


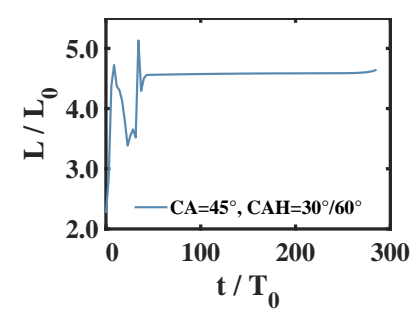

(a)

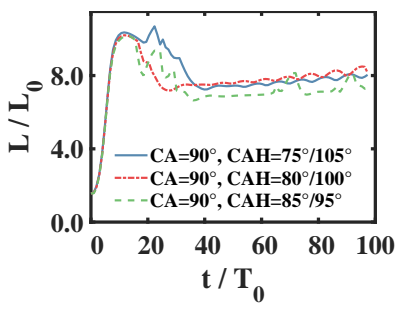

(d)

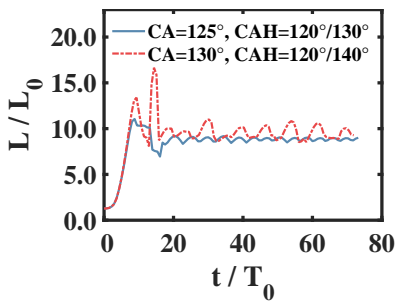

(g)

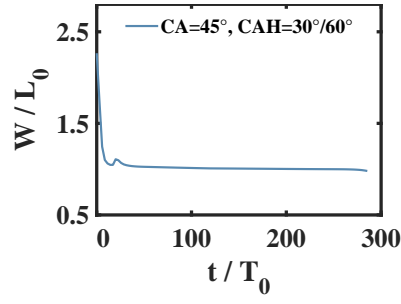

(b)

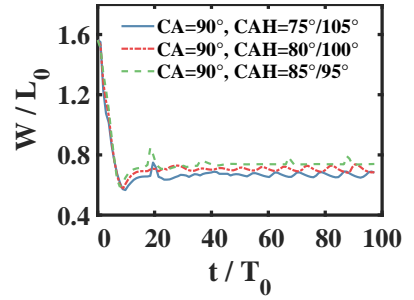

(e)

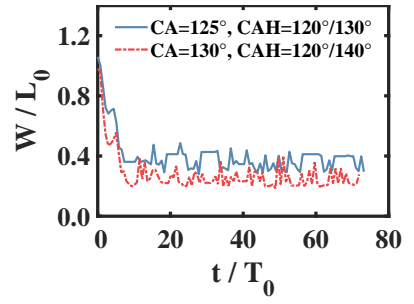

(h)

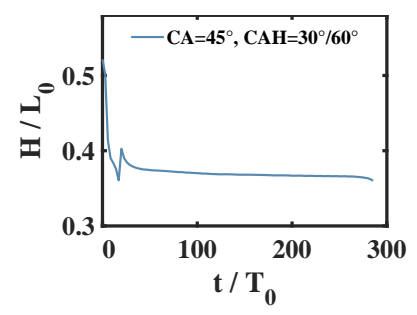

(c)

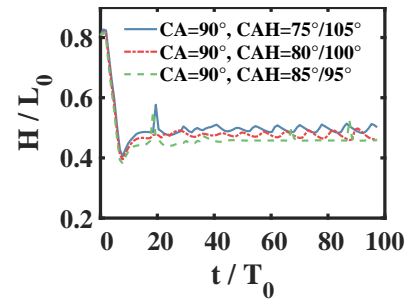

(f)

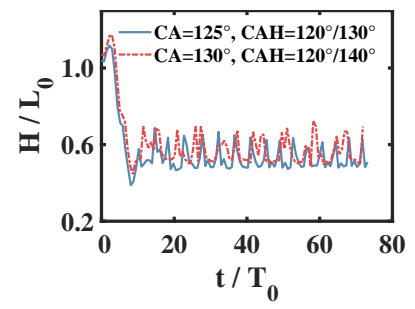

(i)

Figure 11: Morphological analysis for different hysteresis window at $B o=5.0 .(\mathrm{a})(\mathrm{b})(\mathrm{c})$ is the time evolution of normalized length, normalized width, and normalized height in the scope of the hydrophilic hysteresis window, respectively. Similarly, (d)(e)(f) is in the scope of the neutral hysteresis window and $(\mathrm{g})(\mathrm{h})(\mathrm{i})$ is in the scope of the hydrophobic hysteresis window.

wave. After the droplet breakup in the trough of surface wave, the oscillation of length of the droplet will make the downstream part catch up with the upstream part if the oscillation is large enough. Obviously, the degree of oscillation with the neutral hysteresis window can not push the droplet parts to coalesce with each other and thus move forward as separate parts. Finally, the hydrophobic case can have continuous breakup and coalescence.

To complete our analysis, the Bond number, receding angle, and advanc- 


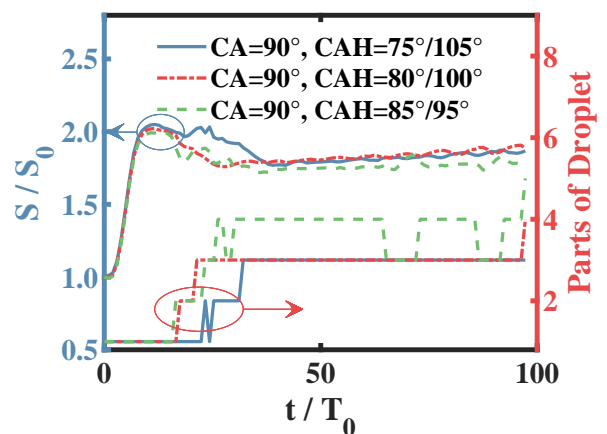

(a) neutral

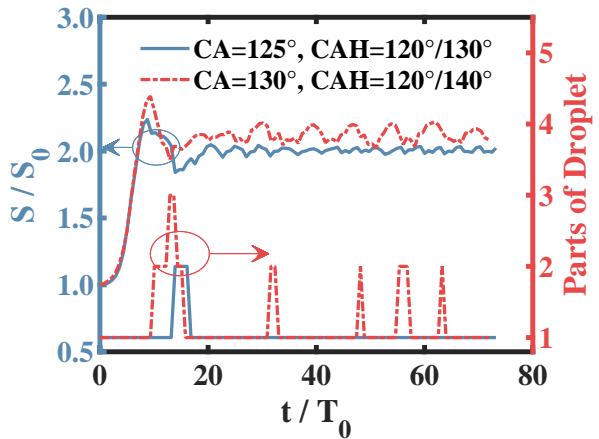

(b) hydrophobic

Figure 12: The surface area and analysis of droplet breakup with varying hysteresis window at $B o=5.0$

ing angle are used as variables for the droplet oscillation transition phase diagram, as shown in Fig. 13. We note that the hydrophobic case keeps coalescing after oscillation occurring while the hydrophilic and neutral cases remain broken up after oscillation occurring.

\section{Conclusion}

In the present study, a modified pseudo-potential LB multiphase model with tunable surface tension is proposed, which is further coupled with the geometrical formulation contact angle scheme to investigate the motion of droplets invoking the contact angle hysteresis. The proposed model can be used to simulate the practical water-air system with large density ratio, good thermodynamic consistency and tunable surface tension. Simulation is conducted to validate the computational model compared with existing literature. Using the validated model, the dynamic behaviour of contact angle hysteresis of droplets for a broad range of the Bond number is investigated on a flat plane. The time evolutions of normalized length, width and height of the droplet are classified into different stages, including stretch, relaxation, and equilibrium. At the equilibrium stage, the oscillation phenomenon of droplet at large Bond numbers is reported for the first time. In addition, it is found that oscillation can lead to the breakup and/or coalescence of the droplet when the surface waves spread on the droplet. To find the deeper mechanism of droplet oscillation, the time evolution of droplet morphology for varying hysteresis window is analysed. Droplets in the hydrophilic hys- 


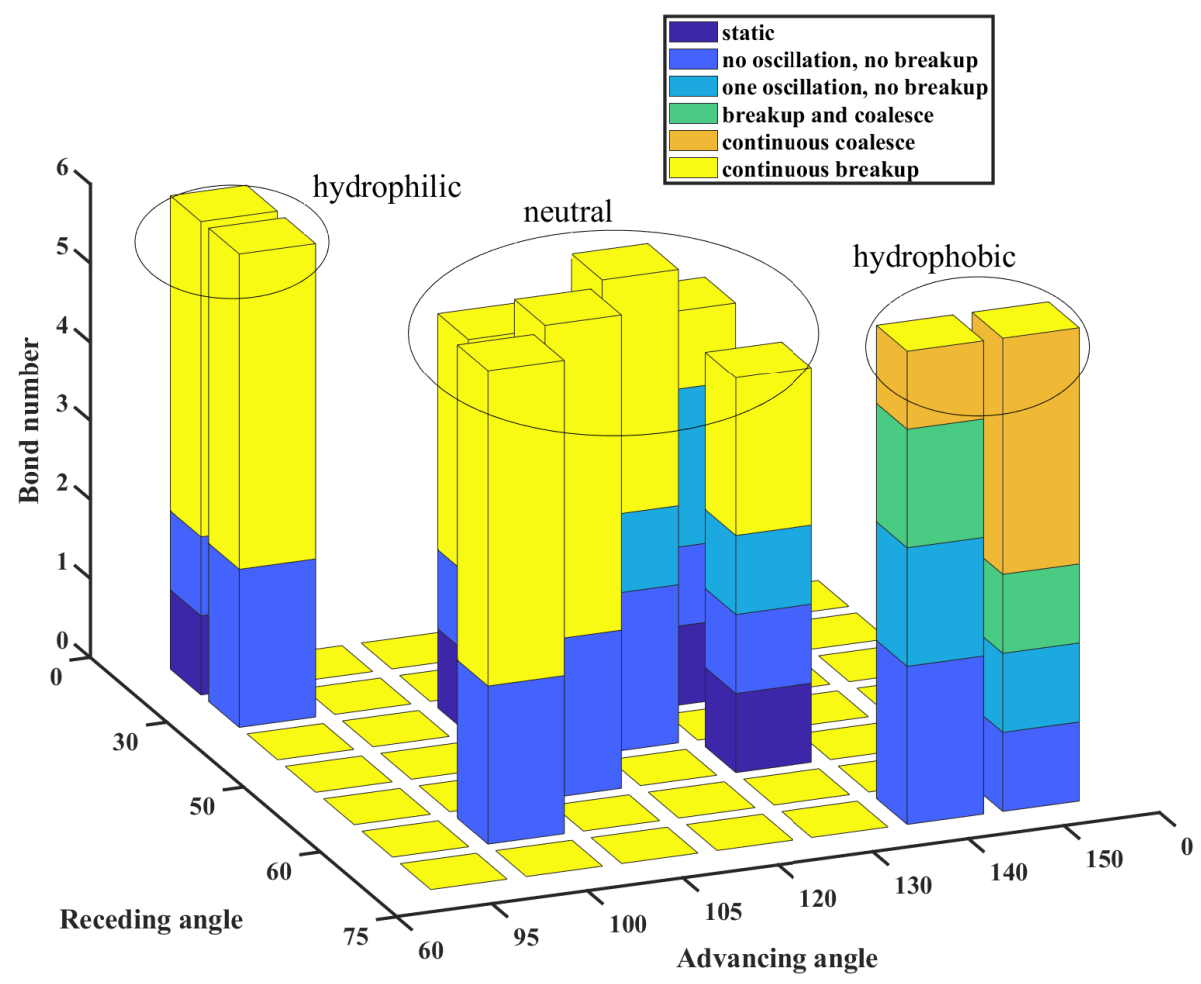

Figure 13: The droplet oscillation transition phase diagram with varying Bond number, receding angle, and advancing angle.

teresis window trend to remain unchanged and no oscillation occurs at the equilibrium stage. For the neutral hysteresis window, there is slight oscillation of the normalized length, width and height at the equilibrium stage while more dramatic oscillation is observed for the hydrophobic hysteresis window. Finally, the phase diagram of droplet oscillation with varying Bond number, receding angle, and advancing angle is obtained. In future, a full phase diagram of droplet oscillation as a function of more influence factors will be created and the phenomena will be created in the laboratory.

\section{Acknowledgements}

This research is supported by the National Key R\&D Program of China (Integration and Control of Fuel Cell Engine for Heavy Duty Truck \& Assessment Technologies of Fuel Cell Engine Reliability for Heavy Duty Truck), the 
Beijing Municipal Science \& Technology Commission (Grant No. Z181100004518004) and the National Key R\&D Program of China (Grant No. 2018YFB0105403). Supercomputing time on ARCHER is provided by the "UK Consortium on Mesoscale Engineering Sciences (UKCOMES)" under the UK Engineering and Physical Sciences Research Council (Grant No. EP/R029598/1).

\section{References}

[1] D. Cwikel, Q. Zhao, C. Liu, X. Su, A. Marmur, Comparing contact angle measurements and surface tension assessments of solid surfaces, Langmuir 26 (19) (2010) 15289-15294. 1

[2] L. Gao, T. J. McCarthy, Wetting 101², Langmuir 25 (24) (2009) 1410514115. 1

[3] H. Eral, J. Oh, et al., Contact angle hysteresis: a review of fundamentals and applications, Colloid and polymer science 291 (2) (2013) 247-260. 1

[4] J. Jovanović, W. Zhou, E. V. Rebrov, T. Nijhuis, V. Hessel, J. C. Schouten, Liquid-liquid slug flow: hydrodynamics and pressure drop, Chemical Engineering Science 66 (1) (2011) 42-54. 1

[5] R. S. Abiev, Effect of contact-angle hysteresis on the pressure drop under slug flow conditions in minichannels and microchannels, Theoretical Foundations of Chemical Engineering 49 (4) (2015) 414-421. 1

[6] C. Fang, C. Hidrovo, F.-m. Wang, J. Eaton, K. Goodson, 3-d numerical simulation of contact angle hysteresis for microscale two phase flow, International Journal of Multiphase Flow 34 (7) (2008) 690-705. 1

[7] X. Zhang, Y. Qin, Contact angle hysteresis of a water droplet on a hydrophobic fuel cell surface, Journal of colloid and interface science 545 (2019) 231-241. 1

[8] S. Wu, M. Ma, A contact angle hysteresis model based on the fractal structure of contact line, Journal of colloid and interface science 505 (2017) 995-1000. 1 
[9] J.-C. Fernández-Toledano, T. Blake, L. Limat, J. De Coninck, A molecular-dynamics study of sliding liquid nanodrops: Dynamic contact angles and the pearling transition, Journal of colloid and interface science 548 (2019) 66-76. 1, 4.1

[10] A. Theodorakakos, T. Ous, M. Gavaises, J. Nouri, N. Nikolopoulos, H. Yanagihara, Dynamics of water droplets detached from porous surfaces of relevance to pem fuel cells, Journal of colloid and interface science 300 (2) (2006) 673-687. 1

[11] M. Santos, J. White, Theory and simulation of angular hysteresis on planar surfaces, Langmuir 27 (24) (2011) 14868-14875. 1

[12] H. Ding, P. D. Spelt, Wetting condition in diffuse interface simulations of contact line motion, Physical Review E 75 (4) (2007) 046708. 1, 2.3

[13] Y. Ba, H. Liu, J. Sun, R. Zheng, Color-gradient lattice boltzmann model for simulating droplet motion with contact-angle hysteresis, Physical Review E 88 (4) (2013) 043306. 11

[14] Q. Li, K. H. Luo, Q. Kang, Y. He, Q. Chen, Q. Liu, Lattice boltzmann methods for multiphase flow and phase-change heat transfer, Progress in Energy and Combustion Science 52 (2016) 62-105. 1

[15] Q. Li, Q. Kang, M. M. Francois, Y. He, K. H. Luo, Lattice boltzmann modeling of boiling heat transfer: The boiling curve and the effects of wettability, International Journal of Heat and Mass Transfer 85 (2015) 787-796. 1

[16] L. Fei, K. H. Luo, C. Lin, Q. Li, Modeling incompressible thermal flows using a central-moments-based lattice boltzmann method, International Journal of Heat and Mass Transfer 120 (2018) 624-634. 1

[17] L. Fei, A. Scagliarini, A. Montessori, M. Lauricella, S. Succi, K. H. Luo, Mesoscopic model for soft flowing systems with tunable viscosity ratio, Physical Review Fluids 3 (10) (2018) 104304. 1

[18] L. Wang, H.-b. Huang, X.-Y. Lu, Scheme for contact angle and its hysteresis in a multiphase lattice boltzmann method, Physical Review E 87 (1) (2013) 013301. 1, 2.3, 2.3 
[19] X. He, S. Chen, R. Zhang, A lattice boltzmann scheme for incompressible multiphase flow and its application in simulation of rayleigh-taylor instability, Journal of Computational Physics 152 (2) (1999) 642-663. 1

[20] T. Lee, L. Liu, Lattice boltzmann simulations of micron-scale drop impact on dry surfaces, Journal of Computational Physics 229 (20) (2010) 8045-8063. 1

[21] H. Liu, Y. Ju, N. Wang, G. Xi, Y. Zhang, Lattice boltzmann modeling of contact angle and its hysteresis in two-phase flow with large viscosity difference, Physical Review E 92 (3) (2015) 033306. 1

[22] H.-Y. Kim, H. J. Lee, B. H. Kang, Sliding of liquid drops down an inclined solid surface, Journal of Colloid and Interface Science 247 (2) (2002) 372-380. 1

[23] C. Semprebon, M. Brinkmann, On the onset of motion of sliding drops, Soft Matter 10 (18) (2014) 3325-3334. 1, 3.1, 3, 3.3, 3.3, 4.1

[24] J.-B. Dupont, D. Legendre, Numerical simulation of static and sliding drop with contact angle hysteresis, Journal of Computational Physics 229 (7) (2010) 2453-2478. 1, 2.3

[25] N. Janardan, M. V. Panchagnula, Effect of the initial conditions on the onset of motion in sessile drops on tilted plates, Colloids and Surfaces A: Physicochemical and Engineering Aspects 456 (2014) 238-245. 1

[26] J. Ziegler, J. H. Snoeijer, J. Eggers, Film transitions of receding contact lines, The European Physical Journal Special Topics 166 (1) (2009) 177180. 1

[27] P. Weidman, S. Krumdieck, P. Rouse, The shape and stability of pinned rotating annular menisci, Journal of Fluid Mechanics 219 (1990) 25-50. 1

[28] K. Katoh, M. Higashine, T. Wakimoto, R. Masuda, On the sliding and profile of a liquid droplet on a rotating disk, Heat Transfer-Asian Research: Co-sponsored by the Society of Chemical Engineers of Japan and the Heat Transfer Division of ASME 39 (1) (2010) 59-75. 1 
[29] D. 't Mannetje, C. Murade, D. Van Den Ende, F. Mugele, Electrically assisted drop sliding on inclined planes, Applied physics letters 98 (1) (2011) 014102. 1

[30] F. Li, F. Mugele, How to make sticky surfaces slippery: Contact angle hysteresis in electrowetting with alternating voltage, Applied Physics Letters 92 (24) (2008) 244108. 1

[31] P. Lallemand, L.-S. Luo, Theory of the lattice boltzmann method: Dispersion, dissipation, isotropy, galilean invariance, and stability, Physical Review E 61 (6) (2000) 6546. 2.1

[32] Q. Li, D. Du, L. Fei, K. H. Luo, Three-dimensional non-orthogonal mrt pseudopotential lattice boltzmann model for multiphase flows, Computers \& Fluids 186 (2019) 128-140. 2.1

[33] L. Fei, J. Du, K. H. Luo, S. Succi, M. Lauricella, A. Montessori, Q. Wang, Modeling realistic multiphase flows using a non-orthogonal multiple-relaxation-time lattice boltzmann method, Physics of Fluids 31 (4) (2019) 042105. 2.1

[34] Z. Guo, C. Zheng, B. Shi, Discrete lattice effects on the forcing term in the lattice boltzmann method, Physical Review E 65 (4) (2002) 046308. 2.1

[35] X. Shan, H. Chen, Lattice boltzmann model for simulating flows with multiple phases and components, Physical Review E 47 (3) (1993) 1815. 2.2

[36] X. Shan, H. Chen, Simulation of nonideal gases and liquid-gas phase transitions by the lattice boltzmann equation, Physical Review E 49 (4) (1994) 2941. 2.2

[37] P. Yuan, L. Schaefer, Equations of state in a lattice boltzmann model, Physics of Fluids 18 (4) (2006) 042101. 2.2

[38] C. E. Colosqui, G. Falcucci, S. Ubertini, S. Succi, Mesoscopic simulation of non-ideal fluids with self-tuning of the equation of state, Soft matter 8 (14) (2012) 3798-3809. 2.2 
[39] Q. Li, K. H. Luo, X. Li, Lattice boltzmann modeling of multiphase flows at large density ratio with an improved pseudopotential model, Physical Review E 87 (5) (2013) 053301. 2.2

[40] D. Zhang, K. Papadikis, S. Gu, Three-dimensional multi-relaxation time lattice-boltzmann model for the drop impact on a dry surface at large density ratio, International Journal of Multiphase Flow 64 (2014) 11-18. 2.2

[41] Q. Li, K. H. Luo, Achieving tunable surface tension in the pseudopotential lattice boltzmann modeling of multiphase flows, Physical Review E 88 (5) (2013) 053307. 2.2, 2.2

[42] N. S. Martys, H. Chen, Simulation of multicomponent fluids in complex three-dimensional geometries by the lattice boltzmann method, Physical review E 53 (1) (1996) 743. 2.3

[43] Q. Kang, D. Zhang, S. Chen, Displacement of a two-dimensional immiscible droplet in a channel, Physics of Fluids 14 (9) (2002) 3203-3214. 2.3

[44] P. Raiskinmäki, A. Koponen, J. Merikoski, J. Timonen, Spreading dynamics of three-dimensional droplets by the lattice-boltzmann method, Computational Materials Science 18 (1) (2000) 7-12. 2.3

[45] R. Benzi, L. Biferale, M. Sbragaglia, S. Succi, F. Toschi, Mesoscopic modeling of a two-phase flow in the presence of boundaries: the contact angle, Physical Review E 74 (2) (2006) 021509. 2.3

[46] Q. Li, K. H. Luo, Q. Kang, Q. Chen, Contact angles in the pseudopotential lattice boltzmann modeling of wetting, Physical Review E 90 (5) (2014) 053301. 2.3

[47] Y. Yu, Q. Li, C. Q. Zhou, P. Zhou, H. Yan, Investigation of droplet evaporation on heterogeneous surfaces using a three-dimensional thermal multiphase lattice boltzmann model, Applied Thermal Engineering 127 (2017) 1346-1354. 2.3

[48] J. Yang, K. H. Luo, X. Ma, Y. Li, S. Shuai, Effects of perforation shapes on water transport in pemfc gas diffusion layers, SAE Technical Papers 2019-April (April) (2019). doi:10.4271/2019-01-0380. 3.1 
[49] J. Yang, X. Ma, T. Lei, K. H. Luo, S. Shuai, Numerical simulations for optimizing the liquid water transport in the gas diffusion layer and gas channels of a pemfc, Journal of Tsinghua University(Science and Technology) 59 (7) (2019) $580-586.3 .1$

[50] P. Perlekar, L. Biferale, M. Sbragaglia, S. Srivastava, F. Toschi, Droplet size distribution in homogeneous isotropic turbulence, Physics of Fluids 24 (6) (2012) 065101. 4.1

[51] M. Wilczek, W. Tewes, S. Engelnkemper, S. V. Gurevich, U. Thiele, Sliding drops: Ensemble statistics from single drop bifurcations, Physical review letters 119 (20) (2017) 204501. 4.1

[52] S. Engelnkemper, M. Wilczek, S. V. Gurevich, U. Thiele, Morphological transitions of sliding drops: Dynamics and bifurcations, Physical Review Fluids 1 (7) (2016) 073901. 4.1

[53] R. J. Donnelly, W. Glaberson, Experiments on the capillary instability of a liquid jet, Proceedings of the Royal Society of London. Series A. Mathematical and Physical Sciences 290 (1423) (1966) 547-556. 4.1 